IJMMS 32:5 (2002) 285-292

PII. S0161171202201210

http://ijmms.hindawi.com

(c) Hindawi Publishing Corp.

\title{
RANDOM SUBGRAPHS OF CERTAIN GRAPH POWERS
}

\author{
LANE CLARK
}

Received 4 March 2002

We determine the limiting probability that a random subgraph of the Cartesian power $K_{a}^{n}$ or of $K_{a, a}^{n}$ is connected.

2000 Mathematics Subject Classification: 05C80.

1. Introduction. A finite, simple, undirected graph $G$ has vertex set $V(G)$ and edge set $E(G)$. The order of $G$ is $|V(G)|$ and the size $e(G)$ of $G$ is $|E(G)|$. For $S \subseteq V(G)$, let $G[S]$ denote the subgraph of $G$ induced by $S$ and $G[S, V(G)-S]$ denote the spanning subgraph of $G$ with edges $x y$ where $x \in S$ and $y \in V(G)-S$. For $U \subseteq V(G)$, let $N_{G}(U)=\{y \in V(G): \exists x y \in E(G)$ with $x \in U\}$ and $\tilde{N}_{G}(U)=N_{G}(U) \cup U$. Of course, $N_{G}(v)=N_{G}(\{v\})$ and the degree $d_{G}(v)$ of $v$ in $G$ is $\left|N_{G}(v)\right|$ for $v \in V(G)$. For $S \subseteq$ $V(G)$, let $b_{G}(S)=|\{x y \in E(G): x \in S, y \in V(G)-S\}|$ and $b_{G}(s)=\min \left\{b_{G}(S): S \subseteq\right.$ $V(G),|S|=s\}(0 \leq s \leq|V(G)|)$.

The Cartesian product $G \square H$ of graphs $G$ and $H$ is the graph with vertex set $V(G) \times$ $V(H)$ where vertices $\left(g_{1}, h_{1}\right)$ and $\left(g_{2}, h_{2}\right)$ are adjacent if and only if $g_{1}=g_{2}$ and $h_{1} h_{2} \in$ $E(H)$, or, $h_{1}=h_{2}$ and $g_{1} g_{2} \in E(G)$. For a graph $G$, define $G^{1}=G$ and $G^{n}=G^{n-1} \square G$ for $n \geq 2$. We use the following recent isoperimetric result of Tillich [6]. Here $K_{a}$ denotes the complete graph of order $a$ and $K_{a, a}$ denotes the complete bipartite graph with parts of order $a$.

LEMMA 1.1 (see [6]). For $G=K_{a}^{n}$ with $a \geq 2$ and $n \geq 1$,

$$
b_{G}(s) \geq(a-1) s\left(n-\log _{a} s\right) \quad \text { for } 1 \leq s \leq a^{n}
$$

and, for $G=K_{a, a}^{n}$ with $a \geq 1$ and $n \geq 1$,

$$
b_{G}(s) \geq \operatorname{as}\left(n-\log _{2 a} s\right) \quad \text { for } 1 \leq s \leq(2 a)^{n} .
$$

Let $G$ be a graph of order $n$ and size $N$. The probability space $\mathscr{G}(G, p)$ consists of all spanning subgraphs $H$ of $G$ where edges of $G$ are chosen for $H$ independently with probability $0 \leq p=p(n) \leq 1$, so that, $\operatorname{Pr}(H)=p^{e(H)} q^{N-e(H)}$ where $q=q(n)=1-p(n)$. (We denote the random graphs in $\mathscr{G}(G, p)$ generally by $G_{p}$.)

In this paper, we determine the limiting probability that $G_{p}$ is connected for $G=K_{a}^{n}$ and $K_{a, a}^{n}$. Specifically, we show that

$$
\lim _{n \rightarrow \infty} \operatorname{Pr}\left(G_{p} \in \mathscr{G}\left(K_{a}^{n}, p\right) \text { is connected }\right)=e^{-\lambda}
$$


for fixed $a \geq 2$ where $p=p(n)=1-q(n)$ with $q(n)=\left[(\lambda(n))^{1 / n} / a\right]^{1 /(a-1)}$ and $\lim _{n \rightarrow \infty} \lambda(n)=\lambda \in(0, \infty)$. In addition, we show that

$$
\lim _{n \rightarrow \infty} \operatorname{Pr}\left(G_{p} \in \mathscr{G}\left(K_{a, a}^{n}, p\right) \text { is connected }\right)=e^{-\lambda}
$$

for fixed $a \geq 1$ where $p=p(n)=1-q(n)$ with $q(n)=\left[(\lambda(n))^{1 / n} / 2 a\right]^{1 / a}$ and $\lim _{n \rightarrow \infty} \lambda(n)=\lambda \in(0, \infty)$. Our first result includes those of Burtin [3], Erdös and Spencer [5], and Bollobás [1] as a special case $(a=2)$. Our approach is similar to [1].

The $r$ th factorial moment of a random variable (r.v.) $X$ is denoted by $E_{r}(X)$. We write $X_{n} \stackrel{d}{\rightarrow} X$ when the sequence $X_{n}$ of r.v.s converges in distribution to the r.v. $X$. Also, we write $P_{\lambda}$ for a r.v. having Poisson distribution with mean $\lambda$.

Let $[n]=\{1, \ldots, n\}$ when $n$ is a positive integer. For a real number $x$ and a positive integer $n$, let $(x)_{0}=1$ and $(x)_{n}=(x) \cdots(x-n+1)$. The cardinality of a set $S$ is denoted by $|S|$. The greatest (least) integer at most (least) the real number $x$ is denoted by $\lfloor x\rfloor(\lceil x\rceil)$. We write $\leq$ for an inequality that holds absolutely for the parameters considered and $\stackrel{*}{\leq}$ for an inequality that holds for the parameters considered and all sufficiently large $n$. We refer the reader to Bollobás [2] for random graph theory and to Durrett [4] for probability.

2. Results. We use the following result from [1].

LEMMA 2.1 (see [1]). If $G$ is a simple graph having order $n \geq 1$, maximum degree $\Delta(G) \leq \Delta$, average degree $d=d(G)=2 e(G) / n$, and $\Delta+1<u<n-\Delta-1$, then there exists a $u$-set $U \subseteq V(G)$ with

$$
\left|\tilde{N}_{G}(U)\right| \geq n \frac{d}{\Delta}\left\{1-\exp \left(-\frac{u(\Delta+1)}{n}\right)\right\} .
$$

Assume $n \geq 2 \Delta+4$, since the result is vacuously true otherwise, and $\Delta>0$ (the righthand side is defined to be 0 for $\Delta=0$ ).

We first consider $G=K_{a}^{n}$ with $V(G)=[a]^{n}$ for fixed $a \geq 2$ and for $n \geq 3$. Note that $V(G)$ is totally ordered lexicographically which naturally extends to $u$-subsets of $V(G)$. In Lemma 2.2 and Theorem 2.5, $\lambda(n)>0$ for all $n$.

LEMMA 2.2. For fixed $a \geq 2, q=q(n)=\left[(\lambda(n))^{1 / n} / a\right]^{1 /(a-1)}$ where $\lim _{n \rightarrow \infty} \lambda(n)=$ $\lambda \in(0, \infty)$, and $p=p(n)=1-q(n)$, we have

$$
\lim _{n \rightarrow \infty} \operatorname{Pr}\left(G_{p} \in G_{(}\left(K_{a}^{n}, p\right) \text { has no isolated vertices }\right)=e^{-\lambda}
$$

Proof. Recall that $G=K_{a}^{n}$. Let $X_{n}\left(G_{p}\right)$ denote the number of isolated vertices in $G_{p}$. Fix $r \in \mathbb{P}$ and let $\mathscr{A}_{r}$ denote the set of $r$-tuples of $V$ with distinct coordinates; $\mathscr{B}_{r}=$ $\left\{\left(v_{1}, \ldots, v_{r}\right) \in \mathscr{A}_{r}: e\left(G\left[\left\{v_{1}, \ldots, v_{r}\right\}\right]\right) \neq 0\right\}$ and $\mathscr{C}_{r}=\mathscr{A}_{r}-\mathscr{B}_{r}=\left\{\left(v_{1}, \ldots, v_{r}\right) \in \mathscr{A}_{r}:\right.$ $\left.e\left(G\left[\left\{v_{1}, \ldots, v_{r}\right\}\right]\right)=0\right\}$. Then $\left|\mathscr{B}_{r}\right| \leq\left(a^{n}\right)_{r-1} r a n \leq a^{n(r-1)} r a n$ and $\left|\mathscr{C}_{r}\right|=\left(a^{n}\right)_{r}-$ $\left|\mathscr{B}_{r}\right| \stackrel{*}{\geq} a^{n r} e^{-r^{2} / a^{n}}-a^{n(r-1)} r a n$. Observe that the number of edges in $G$ incident with $\left\{v_{1}, \ldots, v_{r}\right\}$ is at least $(a-1) r(n-r)$ for all $\left(v_{1}, \ldots, v_{r}\right) \in \mathscr{A}_{r}$. 
First,

$$
\begin{aligned}
0 & \leq \sum_{\left(v_{1}, \ldots, v_{r}\right) \in \mathscr{B}_{r}} \operatorname{Pr}\left(d_{G_{p}}\left(v_{1}\right)=\cdots=d_{G_{p}}\left(v_{r}\right)=0\right) \leq\left|\mathscr{B}_{r}\right| q^{(a-1) r(n-r)} \\
& \leq a^{n(r-1)} \operatorname{ran} \frac{(\lambda(n))^{r-r^{2} / n}}{a^{r(n-r)}}=\frac{(\lambda(n))^{r-r^{2} / n} r a n}{a^{n-r^{2}}} .
\end{aligned}
$$

Next,

$$
\begin{aligned}
\sum_{\left(v_{1}, \ldots, v_{r}\right) \in \mathscr{C}_{r}} \operatorname{Pr}\left(d_{G_{p}}\left(v_{1}\right)\right. & \left.=\cdots=d_{G_{p}}\left(v_{r}\right)=0\right)=\left|\mathscr{b}_{r}\right| q^{(a-1) n r} \\
& \stackrel{*}{\geq}\left[a^{n r} e^{-r^{2} / a^{n}}-a^{n(r-1)} r a n\right] \frac{\lambda^{r}(n)}{a^{n r}} \\
& =\lambda^{r}(n) e^{-r^{2} / a^{n}}-\frac{\lambda^{r}(n) r a n}{a^{n}}
\end{aligned}
$$

while,

$$
\sum_{\left(v_{1}, \ldots, v_{r}\right) \in \mathscr{C}_{r}} \operatorname{Pr}\left(d_{G_{p}}\left(v_{1}\right)=\cdots=d_{G_{p}}\left(v_{r}\right)=0\right) \leq a^{n r} q^{(a-1) n r}=\lambda^{r}(n) .
$$

Hence,

$$
\lambda^{r}(n) e^{-r^{2} / a^{n}}-\frac{\lambda^{r}(n) r a n}{a^{n}} \stackrel{*}{\leq} E_{r}\left(X_{n}\right) \leq \lambda^{r}(n)+\frac{(\lambda(n))^{r-r^{2} / n} r a n}{a^{n-r^{2}}}
$$

so that,

$$
\lim _{n \rightarrow \infty} E_{r}\left(X_{n}\right)=\lambda^{r}
$$

and $X_{n} \stackrel{d}{\rightarrow} P_{\lambda}$ (see [4]).

LEMMA 2.3. For fixed $a \geq 2, q=q(n)=\left[(\ln n)^{1 / n} / a\right]^{1 /(a-1)}$, and $p=p(n)=1-$ $q(n)$, we have

$\operatorname{Pr}\left(G_{p} \in \mathscr{G}\left(K_{a}^{n}, p\right)\right.$ has a component of order $s$ with $\left.2 \leq s \leq a^{n} / 2\right)=o(1)$ as $n \longrightarrow \infty$.

Proof. Recall that $G=K_{a}^{n}$. Let $\mathscr{A}_{s}=\{S \subseteq V(G):|S|=s\}\left(1 \leq s \leq a^{n}\right)$. We consider four cases.

CASE $1\left(2 \leq s \leq s_{1}=\left\lfloor a^{n / 2} / n\right\rfloor\right)$. We have

$$
\begin{aligned}
\mid\left\{S \in \mathscr{A}_{s}: G[S] \text { is connected }\right\} \mid & \leq a^{n} \cdot(a-1) n \cdot 2(a-1) n \cdots(s-1)(a-1) n \\
& \leq a^{n+s} n^{s-1} s^{s},
\end{aligned}
$$

so that (Lemma 1.1)

$$
\begin{aligned}
\sum_{s \in \mathscr{A}_{S}} \operatorname{Pr}\left(G_{p}[S] \text { is a component }\right) & \leq a^{n+s} n^{s-1} s^{s} q^{b_{G}(s)} \\
& =a^{n+s} n^{s-1} s^{s}\left[\frac{(\ln n)^{1 / n}}{a}\right]^{s\left(n-\log _{a} s\right)} \\
& =\frac{1}{n}\left[\frac{a n s^{2} \ln n}{a^{n(1-1 / s)}}\right]^{s} .
\end{aligned}
$$


By examining the derivative $f(s) \ln \left(c e^{2} s^{2} / a^{n}\right)$ with respect to $s$ of $f(s)=c^{s} s^{2 s} / a^{n(s-1)}$ with $c=a n \ln n$, we see that $f(s)$ is decreasing for $s \in\left[2, a^{n / 2} / e c^{1 / 2}\right]$. Here $f(s) \stackrel{*}{\leq}$ $f(2)=16 a^{2} n^{2} \ln ^{2} n / a^{n}$. Hence,

$$
\sum_{s=2}^{s_{1}} \sum_{S \in \mathscr{A}_{S}} \operatorname{Pr}\left(G_{p}[S] \text { is a component }\right) \stackrel{*}{\leq} \sum_{s=2}^{s_{1}} \frac{16 a^{2} n \ln ^{2} n}{a^{n}}=o(1) \text { as } n \rightarrow \infty .
$$

CASE $2\left(s_{1}+1 \leq s \leq s_{3}=\left\lfloor a^{n} / 2\right\rfloor\right)$. Let $\mathscr{B}_{s}=\left\{S \in \mathscr{A}_{s}: b_{G}(S) \geq(a-1) s\left(n-\log _{a}(s / n)\right)\right\}$ and $\mathscr{C}_{s}=\mathscr{A}_{s}-\mathscr{B}_{s}=\left\{S \in \mathscr{A}_{s}: b_{G}(S)<(a-1) s\left(n-\log _{a}(s / n)\right)\right\}$.

First,

$$
\begin{aligned}
\sum_{S \in \mathscr{B}_{S}} \operatorname{Pr}\left(G_{p}[S] \text { is a component }\right) & \leq\left(\begin{array}{c}
a^{n} \\
s
\end{array}\right) q^{(a-1) s\left(n-\log _{a}(s / n)\right)} \\
& \leq\left(\frac{e a^{n}}{s}\right)^{s}\left[\frac{(\ln n)^{1 / n}}{a}\right]^{s\left(n-\log _{a}(s / n)\right)} \\
& =\left[\frac{e(\ln n)^{1-(1 / n) \log _{a}(s / n)}}{n}\right]^{s} \\
& \stackrel{*}{\leq}\left(\frac{e \ln n}{n}\right)^{s} .
\end{aligned}
$$

Hence,

$$
\sum_{s=s_{1}+1}^{s_{3}} \sum_{S \in \mathscr{B}_{S}} \operatorname{Pr}\left(G_{p}[S] \text { is a component }\right) \stackrel{*}{\leq} \sum_{s=s_{1}+1}^{s_{3}}\left(\frac{e \ln n}{n}\right)^{s}=o(1) \quad \text { as } n \longrightarrow \infty .
$$

Next, for $S \in \mathscr{C}_{s}$, let $H=G[S]$. Then

$$
(a-1) s n=\sum_{v \in S} d_{G}(v)=2 e(H)+b_{G}(S)<2 e(H)+(a-1) s\left(n-\log _{a} \frac{s}{n}\right),
$$

so that

$$
2 e(H) \geq(a-1) s \log _{a} \frac{s}{n}
$$

and the average degree $d$ in $H$ satisfies

$$
d>(a-1) \log _{a} \frac{s}{n} .
$$

CASE $3\left(s_{1}+1 \leq s \leq s_{2}=\left\lfloor a^{n} / \ln ^{2} n\right\rfloor\right)$. Let $u=\lfloor s / n\rfloor$, so that $(a-1) n+1 \stackrel{*}{<} u \stackrel{*}{<}$ $s-(a-1) n-1$, and by Lemma 2.1, for sufficiently large $n$, there exists $U \subseteq S,|U|=u$, and

$$
\begin{aligned}
\left|\tilde{N}_{H}(U)\right| & \stackrel{*}{\geq} \log _{a} \frac{s}{n}\left\{1-\exp \left(-\frac{u[(a-1) n+1]}{s}\right)\right\} \\
& \geq \frac{\delta s}{n} \log _{a} \frac{s}{n} \quad \text { with } \delta=1-e^{-1}=0.631 \ldots
\end{aligned}
$$

Let $t=\left\lceil(\delta s / n) \log _{a}(s / n)\right\rceil$, so that $u \stackrel{*}{<} t \stackrel{*}{<} s$, and let $w=s-t=s(1-x)-\tau$ with $x=(\delta / n) \log _{a}(s / n)$ and $0 \leq \tau<1$. Observe that $\delta / 4 \stackrel{*}{\leq} x \stackrel{*}{\leq} \delta$ here. For sufficiently 
large $n$, take the smallest such $u$-set $U=\left\{d_{1}, \ldots, d_{u}\right\}$ in $S(\subseteq V(G))$ which is totally ordered; take the (uniquely determined) first $t-u$ vertices of $\left(N_{G}\left(d_{1}\right) \cap(S-U)\right) \cup$ $\cdots \cup\left(N_{G}\left(d_{u}\right) \cap(S-U)\right)(\subseteq V(G))$; and add the remaining $w$ vertices $W$ of $S$. Then

$$
S \longmapsto\left(\left\{d_{1}, \ldots, d_{u}\right\} ; N_{G}\left(d_{1}\right) \cap(S-U), \ldots, N_{G}\left(d_{u}\right) \cap(S-U) ; W\right)
$$

is an injection. Hence,

$$
\begin{aligned}
\left|\mathscr{b}_{s}\right| & \stackrel{*}{\leq}\left(\begin{array}{c}
a^{n} \\
u
\end{array}\right) 2^{(a-1) n u}\left(\begin{array}{c}
a^{n} \\
w
\end{array}\right) \\
& \leq\left(\frac{e a^{n}}{u}\right)^{u} 2^{(a-1) n u}\left(\frac{e a^{n}}{w}\right)^{w} \\
& \leq\left(\frac{e n a^{n}}{s}\right)^{s / n} 2^{(a-1) s}\left(\frac{e a^{n}}{s(1-x)}\right)^{s(1-x)} .
\end{aligned}
$$

Then (where $x-1 / n \stackrel{*}{>} 0$, Lemma 1.1)

$$
\begin{aligned}
\sum_{s \in \mathscr{C}_{s}} \operatorname{Pr} & \left(G_{p}[S] \text { is a component }\right) \\
& \leq\left|\mathscr{C}_{s}\right| q^{b_{G}(s)} \\
& *\left(\frac{e n a^{n}}{s}\right)^{s / n} 2^{(a-1) s}\left(\frac{e a^{n}}{s(1-x)}\right)^{s(1-x)}\left[\frac{(\ln n)^{1 / n}}{a}\right]^{s\left(n-\log _{a} s\right)} \\
& =\left[(e n)^{1 / n} 2^{a-1}\left(\frac{e}{1-x}\right)^{1-x}\left(\frac{s}{a^{n}}\right)^{x-1 / n}(\ln n)^{1-(1 / n) \log _{a} s}\right]^{s} \\
& *\left[(e n)^{1 / n} 2^{a-1}\left(\frac{e}{1-x}\right)^{1-x}(\ln n)^{1+(2 / n)-2 x-(1 / n) \log _{a} s}\right]^{s} .
\end{aligned}
$$

Here

$$
2 x+\frac{1}{n} \log _{a} s-1-\frac{2}{n} \geq \delta-\frac{1}{2}-\frac{4}{n} \log _{a} n-\frac{2}{n} \stackrel{*}{\geq} \frac{1}{10},
$$

so that

$$
\sum_{S \in \mathscr{C}_{S}} \operatorname{Pr}\left(G_{p}[S] \text { is a component }\right) \stackrel{*}{\leq}\left[(e n)^{1 / n} 2^{a-1}\left(\frac{e}{1-x}\right)^{1-x}(\ln n)^{-0.1}\right]^{S} .
$$

Hence,

$$
\begin{aligned}
\sum_{s=s_{1}+1}^{s_{2}} \sum_{S \in \mathscr{C}_{s}} \operatorname{Pr} & \left(G_{p}[S] \text { is a component }\right) \\
& \stackrel{*}{\leq} \sum_{s=s_{1}+1}^{s_{2}}\left[(e n)^{1 / n} 2^{a-1}\left(\frac{e}{1-x}\right)^{1-x}(\ln n)^{-0.1}\right]^{s} \\
& =o(1) \quad \text { as } n \rightarrow \infty .
\end{aligned}
$$


CASE $4\left(s_{2}+1 \leq s \leq s_{3}\right)$. For $S \in \mathscr{C}_{s}$ and $H=G[S]$, let $T=\left\{v \in S: d_{H}(v) \geq(a-\right.$ 1) $\left.n-\log _{a}^{2} n\right\}, t=|T|$ and $H_{1}=H[T]=G[T]$. Then

$$
\begin{aligned}
2 e\left(H_{1}\right) & =2 e(H)-2 e(H[S-T, T])-2 e(H[S-T]) \\
& >(a-1) s \log _{a} \frac{s}{n}-2(a-1) n(s-t) \\
& =(a-1) s\left[\log _{a} \frac{s}{n}-\frac{2 n}{s}(s-t)\right] .
\end{aligned}
$$

Here

$$
\log _{a} \frac{s}{n} \stackrel{*}{\geq} n-2 \log _{a} n
$$

so that

$$
\begin{aligned}
s(a-1) n-(s-t) \log _{a}^{2} n & \geq \sum_{v \in T} d_{H}(v)+\sum_{v \in S-T} d_{H}(v)>(a-1) s \log _{a} \frac{s}{n} \\
& \geq(a-1) s\left(n-2 \log _{a} n\right),
\end{aligned}
$$

hence,

$$
t \stackrel{*}{\geq} s\left(1-\frac{2(a-1)}{\log _{a} n}\right) .
$$

We take the first $t$ vertices of $T$ for $H_{1}$ where $t=s(1-\epsilon)$ with $s \epsilon=\left\lfloor 2(a-1) s / \log _{a} n\right\rfloor$ so that $0<(a-1) / \log _{a} n \stackrel{*}{<} \epsilon \stackrel{*}{<} 2(a-1) / \log _{a} n \stackrel{*}{<} 1 / 5$. Then

$$
2 e\left(H_{1}\right) \stackrel{*}{>}(a-1) s\left[(1-2 \epsilon) n-2 \log _{a} n\right]
$$

and the average degree $d_{1}$ in $H_{1}$ satisfies

$$
d_{1} \stackrel{*}{>}(a-1)\left[n-\frac{\epsilon}{1-\epsilon} n-\frac{2}{1-\epsilon} \log _{a} n\right] \stackrel{*}{\geq}(a-1)(1-3 \epsilon) n .
$$

Let $u=\left\lceil a^{n} / \ln ^{6} n\right\rceil$, so that $(a-1) n+1 \stackrel{*}{<} u \stackrel{*}{<} t-(a-1) n-1$, and by Lemma 2.1, for all sufficiently large $n$, there exists $U \subseteq T,|U|=u$, and

$$
\begin{aligned}
\left|\tilde{N}_{H}(U)\right| \geq\left|\tilde{N}_{H_{1}}(U)\right| & \stackrel{*}{ } s(1-\epsilon)(1-3 \epsilon)\left\{1-\exp \left(-\frac{u[(a-1) n+1]}{t}\right)\right\} \\
& \geq s(1-\epsilon)^{2}(1-3 \epsilon) \geq s(1-4 \epsilon) .
\end{aligned}
$$

Let $t=s-\lfloor 4 \epsilon s\rfloor$, so that $u \stackrel{*}{<} t \stackrel{*}{<} s$, and $w=\lfloor 4 \epsilon s\rfloor$. For sufficiently large $n$, take the smallest such $u$-set $U=\left\{d_{1}, \ldots, d_{u}\right\}$ in $S(\subseteq V(G))$; take the (uniquely determined) first $t-u$ vertices of $\left(N_{G}\left(d_{1}\right) \cap(S-U)\right) \cup \cdots \cup\left(N_{G}\left(d_{u}\right) \cap(S-U)\right)(\subseteq V(G))$; and add the remaining $w$ vertices $W$ of $S$. Then

$$
S \longmapsto\left(\left\{d_{1}, \ldots, d_{u}\right\} ; N_{G}\left(d_{1}\right)-S, \ldots, N_{G}\left(d_{u}\right)-S ; W\right)
$$


is an injection with $\left|N_{G}\left(d_{i}\right)-S\right| \leq\left\lfloor\log _{a}^{2} n\right\rfloor(1 \leq i \leq u)$. Hence, with $y=\left\lfloor\log _{a}^{2} n\right\rfloor$,

$$
\begin{aligned}
\left|\mathfrak{b}_{s}\right| & \stackrel{*}{\leq}\left(\begin{array}{c}
a^{n} \\
u
\end{array}\right) \sum_{\left(k_{1}, \ldots, k_{u}\right) \in\{0, \ldots, y\} u} \prod_{i=1}^{u}\left(\begin{array}{c}
(a-1) n \\
k_{i}
\end{array}\right)\left(\begin{array}{c}
a^{n} \\
w
\end{array}\right) \\
& \stackrel{*}{\leq}\left(\begin{array}{c}
a^{n} \\
u
\end{array}\right)(y+1)^{u}\left(\begin{array}{c}
(a-1) n \\
y+1
\end{array}\right)^{u}\left(\begin{array}{c}
a^{n} \\
w
\end{array}\right),
\end{aligned}
$$

since

$$
\left(\begin{array}{c}
(a-1) n \\
k
\end{array}\right) \stackrel{*}{\leq}\left(\begin{array}{c}
(a-1) n \\
y+1
\end{array}\right), \quad \forall k \in\{0, \ldots, y\} .
$$

Then

$$
\begin{aligned}
\left|\mathscr{b}_{s}\right| & \leq\left(\frac{e a^{n}}{u}\right)^{u}(y+1)^{u}\left(\frac{e a n}{y+1}\right)^{u(y+1)}\left(\frac{e a^{n}}{w}\right)^{w} \\
& \leq\left(e^{2} a n \ln ^{6} n\right)^{u}\left(\frac{e a n}{\log _{a}^{2} n}\right)^{u y}\left(\frac{e a^{n}}{4 \epsilon s}\right)^{4 \epsilon s} .
\end{aligned}
$$

Hence, (Lemma 1.1)

$$
\begin{aligned}
\sum_{S \in \mathscr{C}_{s}} \operatorname{Pr} & \left(G_{p}[S] \text { is a component }\right) \\
& \leq\left|\mathscr{G}_{s}\right| q^{b_{G}(s)} \\
& \stackrel{*}{\leq}\left(e^{2} a n \ln ^{6} n\right)^{u}\left(\frac{e a n}{\log _{a}^{2} n}\right)^{u y}\left(\frac{e a^{n}}{4 \epsilon s}\right)^{4 \epsilon s}\left[\frac{(\ln n)^{1 / n}}{a}\right]^{s\left(n-\log _{a} s\right)} \\
& =\left[\left(e^{2} a n \ln ^{6} n\right)^{u / s}\left(e a n \ln ^{2} a\right)^{u y / s}\left(\frac{e}{4 \epsilon}\right)^{4 \epsilon}\left(\frac{s}{a^{n}}\right)^{1-4 \epsilon}(\ln n)^{1-(1 / n) \log _{a} s-2 u y / s}\right]^{s} .
\end{aligned}
$$

Here

$$
\begin{gathered}
1 \leq e \operatorname{ean} \ln ^{2} a \stackrel{*}{\leq} e^{2} a n \ln ^{6} n, \quad 0<\frac{u}{s} \stackrel{*}{\leq} \frac{u y}{s} \stackrel{*}{\leq} \frac{5}{\ln ^{2} n}, \\
1-\frac{1}{n} \log _{a} s-\frac{2 u y}{s} \stackrel{*}{\leq} \frac{2}{n} \log _{a} \ln n-\frac{4}{\ln ^{4} n} \stackrel{*}{\leq} 0,
\end{gathered}
$$

so that

$$
\begin{aligned}
\sum_{S \in \mathscr{C}_{S}} \operatorname{Pr}\left(G_{p}[S] \text { is a component }\right) & \stackrel{*}{\leq}\left[\left(e^{3} a^{2} n^{2} \ln ^{2} a \ln ^{6} n\right)^{5 / \ln ^{2} n}\left(\frac{e}{4 \epsilon}\right)^{4 \epsilon} 2^{4 \epsilon-1}\right]^{s} \\
& \stackrel{*}{\leq}\left(\frac{2}{3}\right)^{s},
\end{aligned}
$$

since $\left(e^{3} a^{2} n^{2} \ln ^{2} a \ln ^{6} n\right)^{5 / \ln ^{2} n} \rightarrow 1,(e / 4 \epsilon)^{4 \epsilon} \rightarrow 1$ and $\epsilon \rightarrow 0$ as $n \rightarrow \infty$. Hence,

$$
\sum_{s=s_{2}+1}^{s_{3}} \sum_{S \in \mathscr{C}_{s}} \operatorname{Pr}\left(G_{p}[S] \text { is a component }\right) \stackrel{*}{\leq} \sum_{s=s_{2}+1}^{s_{3}}\left(\frac{2}{3}\right)^{s}=o(1) \text { as } n \longrightarrow \infty .
$$


REMARK 2.4. For all $a \geq 2$ and $n \geq 2, b_{G}(s) \geq 2$ when $2 \leq s \leq a^{n} / 2$. Hence, $0<$ $\tilde{q}(n) \leq q(n)$ implies $(\tilde{q}(n))^{b_{G}(s)} \leq(q(n))^{b_{G}(s)}$ when $2 \leq s \leq a^{n} / 2$. Then (2.10), (2.12), (2.20), and (2.35) hold for $G_{\tilde{p}(n)}$ where $\tilde{p}(n)=1-\tilde{q}(n)$ (the exponent in (2.12) is larger than $b_{G}(s)$ ). Hence, Lemma 2.3 holds for $G_{\tilde{p}(n)}$ as well. The inequalities in the proof of Lemma 2.3 hold for all sufficiently large $n$ which can be determined from nineteen appropriate inequalities there.

THEOREM 2.5. For fixed $a \geq 2, q=q(n)=\left[(\lambda(n))^{1 / n} / a\right]^{1 /(a-1)}$ where $\lim _{n \rightarrow \infty} \lambda(n)=$ $\lambda \in(0, \infty)$, and $p=p(n)=1-q(n)$, we have

$$
\lim _{n \rightarrow \infty} \operatorname{Pr}\left(G_{p} \in \mathscr{G}\left(K_{a}^{n}, p\right) \text { is connected }\right)=e^{-\lambda} .
$$

Proof. We have

$$
\begin{aligned}
0 & \leq \operatorname{Pr}\left(G_{p} \text { is disconnected }\right)-\operatorname{Pr}\left(G_{p} \text { has isolated vertices }\right) \\
& \leq \operatorname{Pr}\left(G_{p} \text { has a component of order } s \text { with } 2 \leq s \leq a^{n / 2}\right)=o(1) \text { as } n \rightarrow \infty,
\end{aligned}
$$

by Remark 2.4. Hence, Lemma 2.2 gives

$$
\lim _{n \rightarrow \infty} \operatorname{Pr}\left(G_{p} \text { is disconnected }\right)=\lim _{n \rightarrow \infty} \operatorname{Pr}\left(G_{p} \text { has isolated vertices }\right)=1-e^{-\lambda} .
$$

We state the result for $G=K_{a, a}^{n}$ since its proof is similar to the proof of Theorem 2.5.

THEOREM 2.6. For fixed $a \geq 1, q=q(n)=\left[(\lambda(n))^{1 / n} / 2 a\right]^{1 / a}$ where $\lim _{n \rightarrow \infty} \lambda(n)=$ $\lambda \in(0, \infty)$, and $p=p(n)=1-q(n)$, we have

$$
\lim _{n \rightarrow \infty} \operatorname{Pr}\left(G_{p} \in \mathscr{G}\left(K_{a, a}^{n}\right) \text { is connected }\right)=e^{-\lambda} .
$$

\section{REFERENCES}

[1] B. Bollobás, The evolution of the cube, Combinatorial Mathematics (Marseille-Luminy, 1981) (C. Berge, D. Bresson, P. Camion, J. F. Maurras, and F. Sterboul, eds.), North-Holland Mathematics Studies, vol. 75, North-Holland, Amsterdam, 1983, pp. 91-97.

[2] _ Random Graphs, 2nd ed., Cambridge Studies in Advanced Mathematics, vol. 73, Cambridge University Press, Cambridge, 2001.

[3] J. D. Burtin, The probability of connectedness of a random subgraph of an n-dimensional cube, Problemy Peredachi Informatsii 13 (1977), no. 2, 90-95.

[4] R. Durrett, Probability. Theory and Examples, The Wadsworth and Brooks/Cole Statistics/Probability Series, Wadsworth and Brooks/Cole Advanced Books and Software, Pacific Grove, California, 1991.

[5] P. Erdös and J. Spencer, Evolution of the n-cube, Comput. Math. Appl. 5 (1979), no. 1, 33-39.

[6] J.-P. Tillich, Edge isoperimetric inequalities for product graphs, Discrete Math. 213 (2000), no. 1-3, 291-320.

LANE CLARK: DEPARTMENT OF MATHEMATICS, SOUTHERN ILLINOIS UNIVERSITY CARBONDALE,

CARBONDALE, IL 62901-4408, USA

E-mail address: 1c1ark@math.siu.edu 


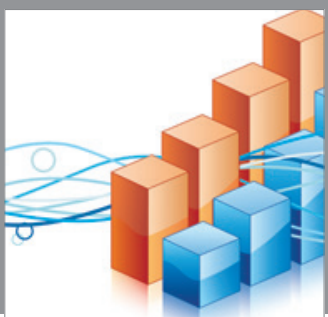

Advances in

Operations Research

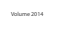

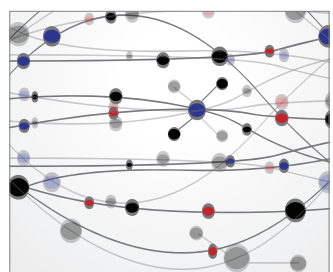

\section{The Scientific} World Journal
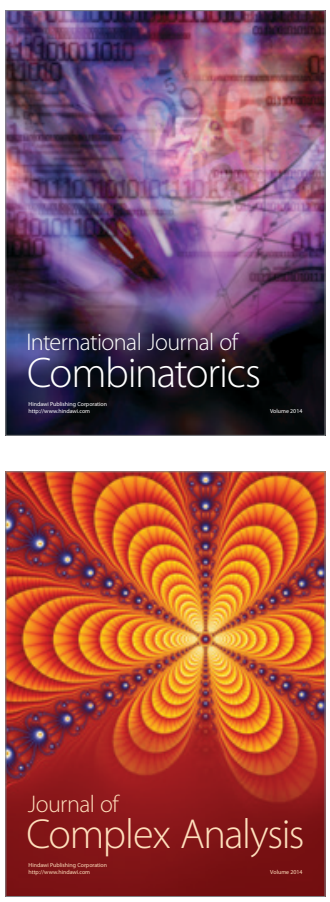

International Journal of

Mathematics and

Mathematical

Sciences
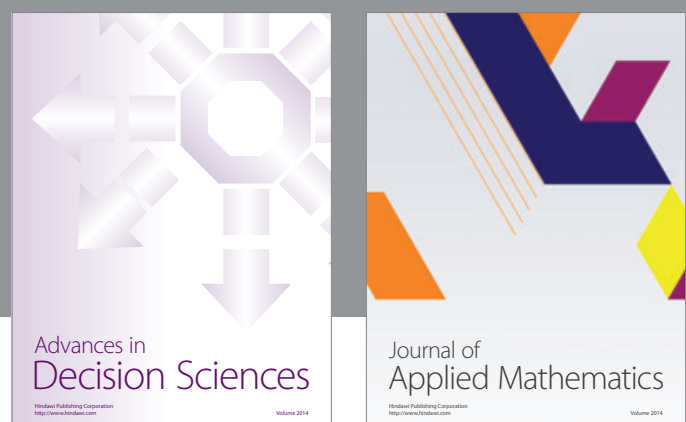

Journal of

Applied Mathematics
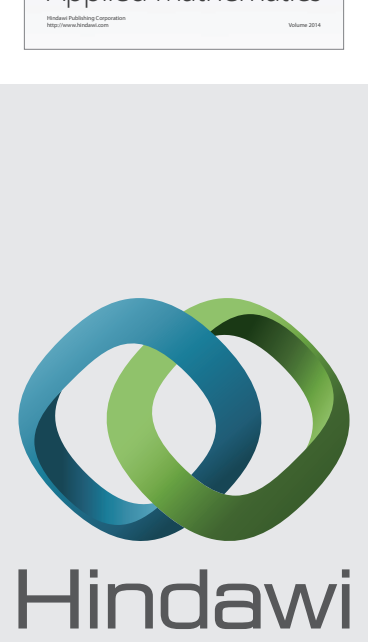

Submit your manuscripts at http://www.hindawi.com
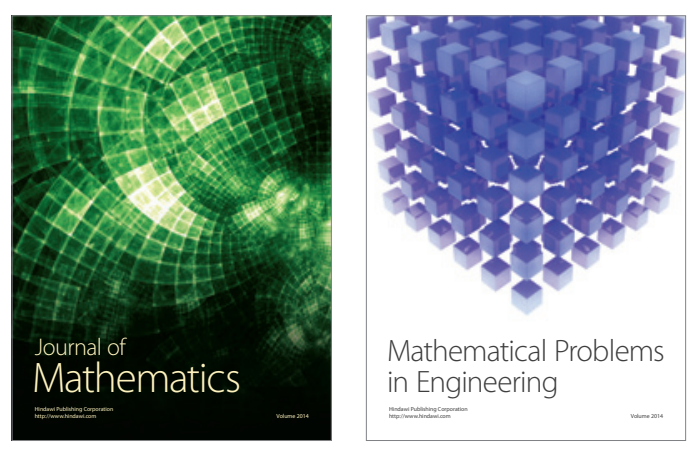

Mathematical Problems in Engineering
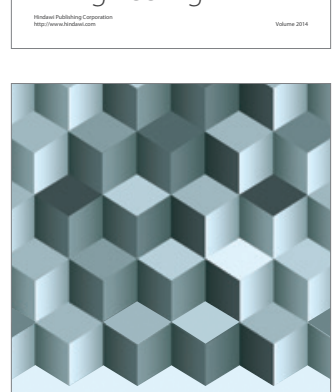

Journal of

Function Spaces
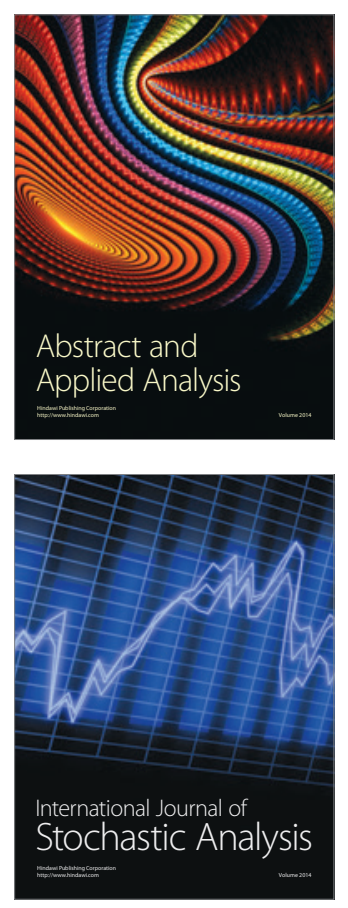

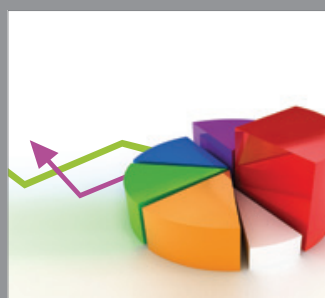

ournal of

Probability and Statistics

Promensencen
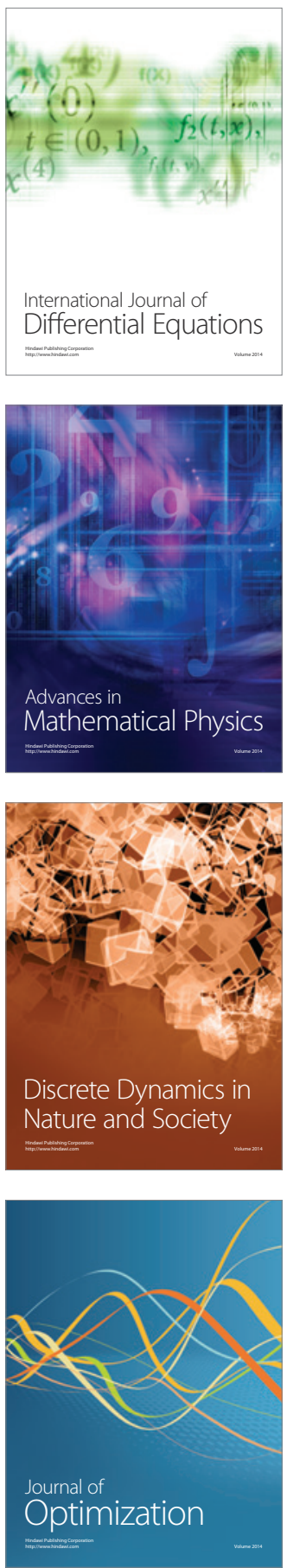